\title{
Analysis on Special Training Methods of Track and Field Sprint
}

\author{
Yanmin Zhao
}

\author{
Huazhong Agricultural University, Wuhan, Hubei 430070
}

\section{Keywords: Track and field sprint; Special training; Method analysis}

\begin{abstract}
Sprint is a basic project of track and field sports, and it usually includes 50m-run, 60m-run, 100m-run, 200m-run, 400-run as well as 4×100 and 4×400 delay, etc. Athletes are required to use the complete running of the prescribed distance in the determined runway at the fastest speed, and the first to get to terminal point is the winner. In terms of human body function, the track and field sprint can effectively develop athletes' speed and quality and maximize human instinct. It is also a sport that needs long-term exercise, so athletes of track and field run need to master certain methods and skills so as to achieve the effect of training. This paper will especially analyze the methods for training track and field sprint.

Sprint is a type of exercise that requires extreme strength that athletes are required to complete the required distance at the fastest speed. Human bodies and organs should complete the largest amount of exercises under hypoxic conditions. In order to achieve good results in track and field sprint, special and scientific training methods are required. According to the training methods and usage, sprint can be divided into many categories and each category has different training methods, different sprints should have different training methods, so athletes are required to truly master the training contents and training methods and apply them into training so as to effectively improve their own sports skills and prove themselves in actual competition. The training of sprint training techniques and methods is an integral whole and is mainly composed of acceleration run, on-the-way run and terminal run.
\end{abstract}

\section{Training of Athletes' Physical Quality}

Training of physical quality is a necessary condition to improve the sprint performance. As qualified sprint athlete must meet the requirements for some qualities, such as speed, strength, durance and relatively strong flexibility, so it is necessary to pay attention to methods while training physical quality in sprint, in which the speed is the main determining factor of an athlete's performance. How to improve sprint athletes' speed is also a concern for every trainer. Of course, special training of speed cannot be separated from special training of strength and technical support.

Training of Lower Limbs' Comprehensive Quality. Chest-touch jump. Open the two legs to the same width as shoulders, and then swing the arm upwards to drive the body to jump, and then curl abdomen and feet. Repeatedly practice this, because this is the key point of strengthening waist and abdominal strength.

Frog jump. Don't need to squat frog, but to semi-squat frog. And continue to jump without stopping in the middle, the general jump 20-30 meters; this can be very effective to enhance the thigh endurance and basic strength.

Jump the steps or stairs. In the jump when we are trained to wear knee pads, this can not only enhance the thigh explosive force can also strengthen the projectile velocity.

We can also enhance the toughness and strength of ankle joint by stepping tip, and long-term relax tense muscles.

High leg training load. You can find a barbell weight or other things, high leg fast, increase the comprehensive ability of thigh.

Run after training. You can find the wall or by using the parallel bars, with his hands on them, the body and the ground 45-60, through the rapid exchange of leg, in the process to keep the legs should be straight, and then lift the leg to go.

Training variable speed running. Generally, we need to run 600-800 meters, straight running at full speed, running in the corner or walking can be ok. 
Exercises to Develop Endurance. The good endurance quality is the premise of the athlete's technology. In the training, if the athlete's endurance quality is poor, then in the back of the running stage, the overall speed of the athletes will fall, the action will be deformed. Therefore, it is very important to develop the endurance quality of the athletes.

There are three main methods to train endurance: one is the general endurance, which is trained through cross-country race; the second is speed endurance, which is main trained through sprint in order to keep the fastest speed within a certain period of time while repeatedly combining long-distance race; the third is strength endurance, which is to improve the complete support for sprint athletes in fast-speed running. Effective way to practice the power of endurance is the weight of continuous jump, long jump, high jump, stride leg lift and exercise method using single foot.

Training to Develop Strength. Good strength is the main power to push forward the human body, so it is very important to develop the special strength quality of sprint athletes in training of sprint. In the training of sprinters strength training, should be through the use of the upper limb and body weight of lower extremity strength training and strength training, such as through a small dumbbell to arm exercises, or lifting barbell squat and other exercises every week, in practice to combine the power of dynamic exercises such as fast running or fast swing the arm exercises. In the development of Special Forces in the process must be combined with special technology, only a good special technology to effectively play a special force, the power will be converted to speed.

\section{Methods of Training Athletes' Coordination, Sensitivity and Reaction Ability}

Training of Coordination and Sensibility. The body's coordination and sensitivity play an important role in sprint-training. In order to run faster, we must have good coordination to power and high sensitivity. To train the coordination degree and sensitivity of the athletes by coordinating the running or jumping exercises.

The practice method of coordinated running and jumping exercises, coordination is mainly run small step to run, run and run high leg kicking and other special training with different combination of different directions, like running pace and swing arm running practice field. Jumping exercise is mainly double squat jump, double jump, double jump rope relay race, and some other practice methods.

Training of Flexibility. Flexibility refers to the range of activities of the various joints of the exercise, is the extension of the muscle ligament exercises. It must have athletes in sprint, plays an important role in increasing the pace of athletes. In training or kicking is often used pressure leg contact with enhanced flexibility. Flexible quality refers to the extent of the various joint activities, the extension of the muscle ligament. It plays an important role in the sprint movement. The following methods are usually adopted in general training: (1)good morning exercise(2)Pull the lever leg(3)Vertical and horizontal fork(4)kick legs from the positive side and external sides(5)Quick squat exercise.

Training of Reaction Ability. Good reaction ability is a necessary condition for sprint. The speed of reaction and the quick start of the start of the sprint can improve the speed of the sprint race. Improve the speed of the reaction of athletes, should focus on improving his ability to sense of hearing. Through these training methods, such as a sudden signal, listening to gunfire, clapping and whistling sound, also can make athletes do quick start, turn back to sprint, starting, or fast running in place high leg after a few seconds after hearing the signal, which can improve the response speed of athletes.

\section{Training Sprint Athletes' Skills}

Special Technical Training. We must make full use of the principle of the human body movement and the skills of the project, to give full play to the potential of the human body, a reasonable and effective completion of special sports. In the process of training of sprint, is composed of a series of techniques for sports, a complete set of special technology is composed of technical basis, technical process and technical details, but also has the obvious individual. Whether athletes have a good 
command of reasonable and correct technical action is the basic condition for the future to achieve high level. Dash technology is the main way to improve sprint performance of sprint athletes. In order to improve the sprint technology, it is necessary to carry out the training of all aspects of the sprint technology and running special action.

Methods that can effectively improve sprint athletes' speed in training include: (1)Intermittent running, each run to achieve maximum speed, thereby improving the ability and speed of the maximum speed. For example, in the 30 meters, 60 meters and 100 meters' short intervals in practice, because the number of short distance running high strength must be in practice the pulse recovery before the next practice, so that students can be fully run in every practice. (2) Repeats, athletes in the repeated run, to run fast or medium run, in 100 meters, 150 meters, 200 meters and 400 meters in practice by continuously adjusting the breathing, ensure run when no deformation technology. (3) Variable speed run, in the training process by jogging combination, in order to develop the athlete's speed and ability of aerobic metabolism, usually by 50 meters +50 meters run jogging way, each exercise 10 times for a group. (4)Combination of repeated running, mainly from long to short, from short to long and other forms of exercise. In training for students to use from the long to short practice, such as 200 meters +100 meters +50 meters of training, to practice 3,4 groups, through this to improve the speed of endurance athletes. (5)et distance run, it is at the start of the first to let each other after a certain distance, and then go after. In the training, can make a good athlete sprint performance let poor athletes start at a distance, and then by the sprint athletes with good recovery. This training to accelerate the ability of students and to a certain extent, to encourage athletes to go all out to the determination of athletes. (6)Accelerating speed running, which can be exercised by even-speed + running-maximum-speed running + slow speed running + accelerating running, etc. General training distance is 80-100 meters, the conversion stage in practice, can use the whistle, this can improve the ability to accelerate the athletes and the ability to relax.

Tactical Training of Sprint. The track and field sprint needs to match certain tactics, so the training of the effective training of the athletes can improve the performance of the athletes in the competition. Athletes in the game to understand each other's strength and external conditions, so as to give full play to their ability. In the track and field sprint race in the main tactics: first of all, the team players need to save the strength, in the final or final sprint to go all out, so as to win the better results. Secondly, we should also play the spirit of unity and cooperation, so that we can cooperate with each other.

\section{Effective Suggestions of Training Sprint}

According to the practical theory and practice training, combined with the sprint training for scientific training methods. Fully according to the physical fitness of athletes training intensity and carry out the exercise mode, pay attention to the development of special technology for athletes, for their individual differences in treatment.

Training Means to Improve Speed. High speed and large swing legs before and after the swing exercise, the requirements of the rapid swing in the completion of the folding technology of nursing, swing legs and legs folded more tightly, the smaller the radius, the faster the swing.

To speed up the foot speed exercise requirements as much as possible to shorten the flight time.

Quickly swing arm and legs, leg arm movement in coordination.

Training Method to Improve Speed. (1) $20-40 \mathrm{~m}$ quick running exercise(2) $4 \times 25-50 \mathrm{~m}$ relay, accelerating running and chasing-running exercise (3)downhill running exercise; (4)run with the wind; (5)Variable speed running exercises for all kinds of short distance.

Training to Enhance Athletes' Psychological Quality. In the track and field sports competition, should pay attention to the athlete's psychological quality of the suspension chain, a poor psychological quality of the athletes, he even if the technical level is high, it is impossible to achieve good results in the field. Therefore, in general training to strengthen the training of the 
psychological quality of the athletes. For example, you can make self-suggestion and relaxation training, athletes need to relax after a lot of exercise training to eliminate fatigue, but also in the game before the excessive excitement also need to relax to calm their emotions. The content and methods of special mental suspension used in sprint-training are: (1)To strengthen the formation and development of personality psychological characteristics of sprint athletes, so that athletes can regard the sprint as a science; (2)Sprint racing simulation training, in order to let the players understand the game, predict the various unfavorable factors, improve the ability to adapt to the psychological quality, give full play to the level of sprint. The main psychological quality and training of sprint athletes is the training and competition in the training and competition in the training of sprint athletes in the winter, self-confidence and attention; (3)training and cultivation of pre-competition mental state. Only by making full preparation before the competition can we create excellent result. Therefore, it is very important for coaches to train and cultivate sprint athletes' pre-competition mental state.

\section{Conclusion}

Sprint is a sport familiar for everyone. Exercise of sprint can exercise and cultivate a strong will, improve the function of human organs, effectively promote the growth and development of human body and enhance physical fitness. Therefore, in sprint-training, it is necessary to carry out comprehensive and systematic training, because only after repeated training can athletes have the necessary skills and psychological quality. Coaches should constantly change the training methods according to athletes' own features and the training conditions, innovate training methods so as to improve athletes' ability to adapt, reach the best effect of starting so as to obtain excellent results.

\section{References}

[1] Mikami,E., Fuku,N., Murakami, H. et al.ACTN3 R577X genotype is associated with sprinting in elite Japanese athletes[J].International journal of sports medicine,2014,35(2):172-177.

[2] Simon,Y.,Kessler,S.M.,Bohle,R.M. et al. The insulin-like growth factor 2 (IGF2) mRNA-binding protein p62/IGF2BP2-2 as a promoter of NAFLD and HCC?[J].Gut: Journal of the British Society of Gastroenterology,2014,63(5):861-863.

[3] Bret,C.,Lacour,J.-R.,Bourdin, M. et al. Differences in lactate exchange and removal abilities between high-level African and Caucasian 400-m track runners[J].European journal of applied physiology,2013,113(6):1489-1498.

[4] Kazuyoshi Miyaguchi, Shinich Demura, Kazuya Nagai, et al.Comparison of base running in baseball players and track-and-field athletes[J].Health,2012,03(01):26-31.

[5] Benito-MARTíNEZ,E.,Martínez-Amat,A.,Lara-SáNCHEZ,A.J. et al.Effect of combined electrostimulation and plyometric training on 30 meters dash and triple jump[J].The Journal of sports medicine and physical fitness,2013,53(4):387-395.

[6] Valente EA, Sheehy ME, Avila JJ, et al. The effect of the addition of resistance training to a dietary education intervention on Apo lipoproteins and diet quality in overweight and obese older adults[J].Clinical Interventions in Aging,2011,2011(default),6(5):235-261.

[7] Análisis del rendimiento en competición entre corredores de 100 metros lisos de diferente nivel. (Performance analysis in competition between athletes 100 meters dash athletes of different levels).[J].RICYDE. Revista Internacional de Ciencias del Deporte, 2011, 7(26).

[8] Beck, W.C., Holzman, M.D., Sharp, K.W. et al.Comparative effectiveness of dynamic abdominal Sonography for hernia vs computed tomography in the diagnosis of incisional hernia[J].Journal of the American College of Surgeons,2013,216(3):447-453.

[9] Andersen,C.H.,Andersen,L.L.,Gram,B. et al.Influence of frequency and duration of strength 
training for effective management of neck and shoulder pain: A randomised controlled trial[J].British journal of sports medicine,2012,46(14):1004-1010.

[10] Eric L. Sauers,Danelle L. Dykstra,R. Curtis Bay et al.Upper Extremity Injury History, Current Pain Rating, and Health-Related Quality of Life in Female Softball Pitchers[J].Journal of sport rehabilitation,2011,20(1):100-114. 